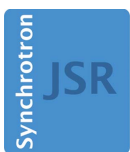

JOURNAL OF SYNCHROTRON RADIATION

ISSN 1600-5775

Received 3 August 2018

Accepted 14 November 2018

Edited by Y. Amemiya, University of Tokyo, Japan

Keywords: X-ray diffuse scattering; diffraction; inelastic X-ray scattering; phonons; disorder.

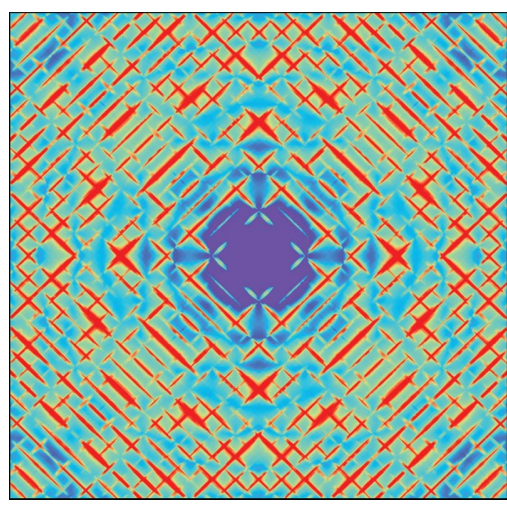

OPEN $\odot$ ACCESS

\section{A new diffractometer for diffuse scattering studies on the ID28 beamline at the ESRF}

\author{
A. Girard, ${ }^{a *}$ T. Nguyen-Thanh, ${ }^{b}$ S. M. Souliou, ${ }^{b}$ M. Stekiel, ${ }^{a}$ W. Morgenroth, \\ L. Paolasini, ${ }^{\text {b }}$ A. Minelli, ${ }^{\text {b }}$ D. Gambetti, ${ }^{b}$ B. Winkler ${ }^{\mathrm{a}}$ and A. Bosak ${ }^{\text {b* }}$ \\ anstitut für Geowissenschaften, Goethe Universität Frankfurt, Altenhöferallee 1, D-60438 Frankfurt am Main, \\ Germany, and ${ }^{\mathbf{b}}$ ESRF - The European Synchrotron, 71 Avenue des Martyrs, F-38000 Grenoble, France. \\ *Correspondence e-mail: adrien.girard@sorbonne-universite.fr, bossak@esrf.fr
}

A new diffractometer is now available to the general user community at the ESRF. The new diffractometer is a side station of the high-resolution inelastic X-ray scattering spectrometer on beamline ID28 and is located in the same experimental hutch. Both instruments can be operated simultaneously. The new diffractometer combines a fast and low-noise hybrid pixel detector with a variable diffraction geometry. The beam spot on the sample is $50 \mu \mathrm{m} \times 50 \mu \mathrm{m}$, where focusing is achieved by a combination of Be lenses and a $\mathrm{KB}$ mirror. Wavelengths from 0.5 to $0.8 \AA$ can be used for the diffraction experiments. The setup is compatible with a variety of sample environments, allowing studies under non-ambient conditions. The diffractometer is optimized to allow a rapid survey of reciprocal space and diffuse scattering for the identification of regions of interest for subsequent inelastic scattering studies, but can also be employed as a fully independent station for structural studies from both powder and single-crystal diffraction experiments. Several software packages for the transformation and visualization of diffraction data are available. An analysis of data collected with the new diffractometer shows that the ID28 side station is a state-of-the-art instrument for structural investigations using diffraction and diffuse scattering experiments.

\section{Introduction}

X-ray diffraction is one of the most used experimental techniques to determine structural parameters. While in conventional X-ray diffraction experiments the focus is on the determination of the location and intensities due to Bragg scattering, it is well known that an analysis of the intensity distribution of diffuse scattering can provide additional information due to static or dynamic deviations from the longrange-ordered average structure (Bosak et al., 2015; Wehinger, Chernyshov et al., 2014; Wehinger, Bosak et al., 2014; Wehinger et al., 2015; Welberry, 1985). While static short-range order or the presence of defects also cause diffuse scattering at very low temperatures, analysis of the diffuse scattering which shows a strong temperature dependence, the so-called 'thermal diffuse scattering' (TDS), is a unique tool to probe dynamic aspects of the system under study, including the dispersion of low-frequency phonons and consequently also the elastic behaviour of crystals. The instrument introduced here is optimized for diffuse scattering studies.

TDS studies are particularly efficient when coupled with spectroscopic investigations and state-of-the-art ab initio calculations; see Bosak et al. (2015) for a review. Specifically, a fast mapping of reciprocal space prior to an inelastic X-ray scattering (IXS) experiment allows the identification of regions of interest, which can then be explored in more detail 
by energy-resolved spectroscopic measurements. The need for a fast method to localize regions of interest in reciprocal space prior to an IXS experiment, which involves long counting times, was the main motivation for the design and construction of a side station to the inelastic high-resolution spectrometer on beamline ID28.

By design, the diffractometer was planned to complement high-energy-resolution IXS measurements and therefore was located in the experimental hutch of ID28. An exisiting Huber diffractometer was equipped with a state-of-the-art hybrid pixel detector. Parallel operation of the diffractometer and spectrometer was a design goal. The synchrotron radiation used for the diffraction experiments is obtained by diverting a small portion of the beam used by the high-resolution spectrometer to the side station, and the setup allows independent operation of the two instruments.

The diffractometer has been operational and open to the general user community since 2017. In its first year of operation, it has met all design specifications and has been employed in studies of diffuse scattering in a large class of materials ranging from strongly correlated electron systems to nanoscale-modulated and low-dimensional systems. Most investigations have benefited from the broad range of available sample environments. The diffuse scattering diffractometer, in combination with the existing high-resolution IXS instrument on ID28, constitutes a globally unique experimental station, offering unprecedented capabilities in the study of lattice dynamics in condensed matter and of the real structure of functional materials to the large ESRF user community.

\section{Beamline overview}

The ID28 beamline (Fig. 1) is primarily dedicated to momentum-resolved high-energy-resolution inelastic X-ray scattering with millielectronvolt energy resolution. With such IXS measurements the collective motions of atoms can be probed as a function of wavevector transfer. In crystalline materials, the determination of phonon dispersion by IXS provides insight into the origin of phase transitions (Hoesch et al., 2009; Weng et al., 2014), and can be used to determine elastic and thermodynamic properties (Antonangeli et al., 2008; Lethbridge et al., 2009) and to understand the interplay between electronic, magnetic and lattice degrees of freedom (Souliou et al., 2016; Le Tacon et al., 2013; Rueff et al., 2002). In disordered matter, IXS allows the nature of sound propagation and relaxation mechanisms at terahertz frequencies to be addressed (Niss et al., 2008; Damart et al., 2015; Bencivenga et al., 2007). A spectral line-shape analysis gives direct insight into relevant physical parameters such as viscosity, relaxation time, and elastic and viscous sound speeds (Niss et al., 2008; Pogna et al., 2015; Izzo et al., 2011).

The main applications of IXS comprise studies of strongly correlated electron systems [superconductors (Walters et al., 2015; Zocco et al., 2015; d'Astuto et al., 2013), charge-density wave systems (Leroux et al., 2015; Le Tacon et al., 2013), lanthanides and actinides], complex and functional materials (clathrates, zeolites, relaxor and conventional ferroelectrics and ferroics, large-band-gap semiconductors) (Borissenko et al., 2013; Gvasaliya et al., 2012; Lortz et al., 2008; Lethbridge et al., 2009), minerals of relevance in earth and planetary science

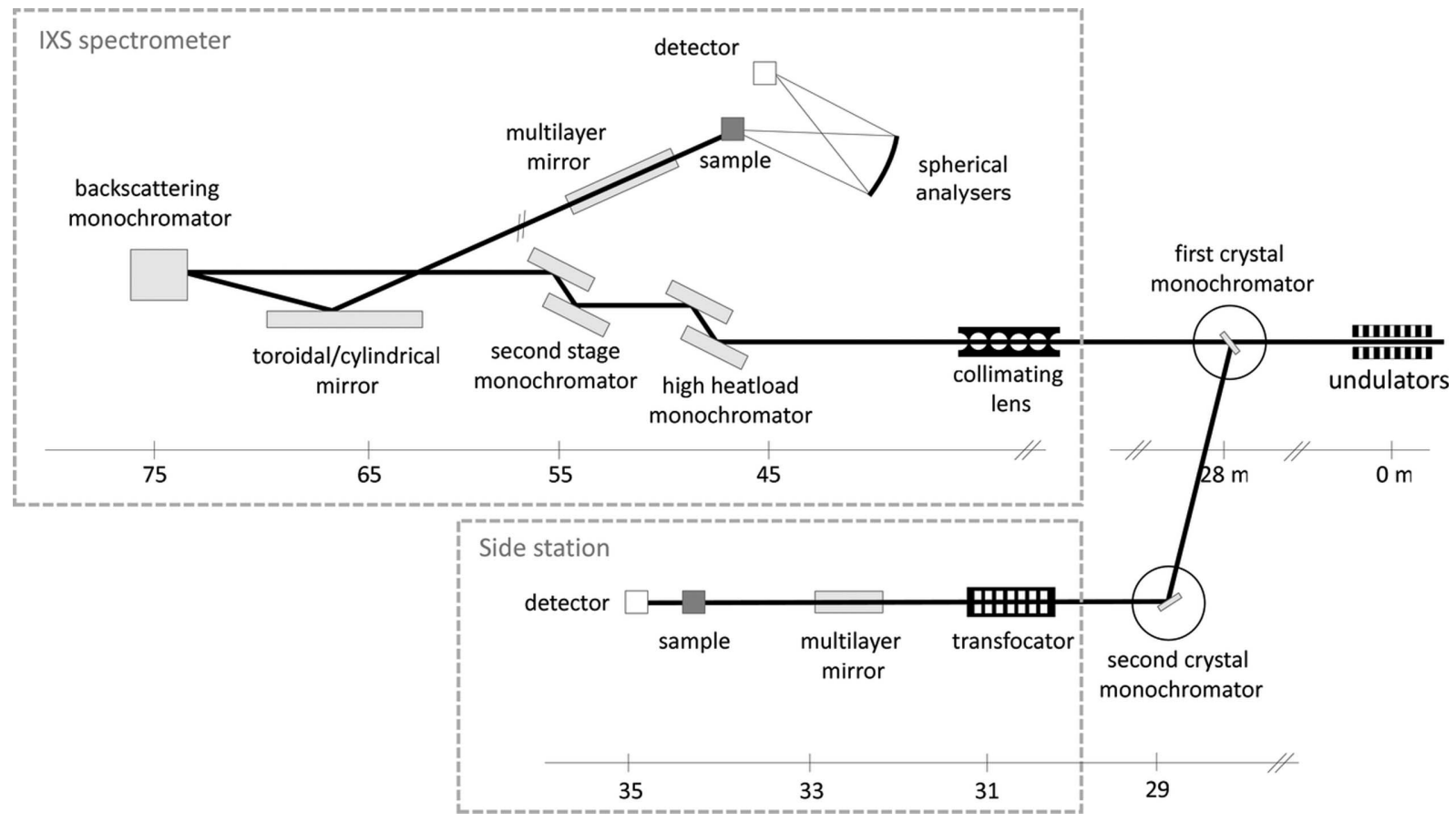

Figure 1

Schematic layout of the inelastic scattering beamline ID28 at the ESRF, showing (top) the IXS spectrometer, (bottom) the side station and (upstream) the first crystal of the double-crystal monochromator splitting the beam in two. 
(Antonangeli et al., 2010, 2011; Fiquet et al., 2009), and liquids and glasses (Niss et al., 2008; Pogna et al., 2015; Izzo et al., 2011).

However, IXS studies require a substantial amount of beam time (typically days) and are not well suited to surveying reciprocal space. Instead, in order to carry out IXS measurements efficiently, prior information with respect to an optimal choice of energy and momentum transfer is required. While state-of-the-art ab initio calculations can often provide such information, they are typically restricted to the harmonic approximation in the athermal limit. As most phenomena of interest are either strongly temperature dependent or are anharmonic, an alternative is required to constrain the regions of reciprocal space which are of interest. Here, diffuse scattering studies are an ideal tool, as they can be carried out in minutes.

\subsection{Beamline design}

The conceptual scheme of the side station is based on the requirement for parallel operation of the diffractometer with the main branch of the ID28 beamline, the IXS spectrometer. The ID28 straight section is currently equipped with a revolver of three undulators of either $32 \mathrm{~mm}$ or $17 \mathrm{~mm}$ magnetic period (U32/U17). The choice of the magnetic period is the result of a compromise between the necessary tunability within the working energy range between 13.8 and $25.7 \mathrm{keV}$ and maximizing the flux by reducing the undulator period as much as possible. In order to extract a useful beam for the diffractometer, a Laue-Bragg monochromator (LBM) is used with the (311) (Laue) reflection from the first (splitter) diamond crystal and the (422) (Bragg) reflection from the second silicon crystal. The first crystal is water cooled and the second is placed in a He-filled chamber without forced cooling [see Figs. 2(a) and 2(b)].

The second Si crystal of the monochromator is mounted on a translation stage nearly parallel to the main beam direction, to follow the beam-path change after the first crystal. The general layout of the double-crystal monochromator and the side station is shown in the bottom part of Fig. 1. The monochromator allows the instrument to be operated in the energy range $12.6-23.7 \mathrm{keV}$. The side station typically operates on the same undulator harmonic as the main branch of ID28, except in low-current four-bunch filling mode, where an unrestricted choice of wavelength is available. Nevertheless, if a specific energy is required, it is possible to operate the station with beam from one undulator while using the radiation from the two others for the IXS station.

Further optical elements and the diffractometer are located in experimental hutch EH1, next to the IXS spectrometer. The $\mathrm{X}$-ray beam is first focused by a transfocator equipped with cylindrical Be refractive lenses in the vertical direction and a multilayer mirror in the horizontal direction. Such a scheme minimizes the optics and diffractometer adjustments required during a photon energy change, permits easy variation of the focal shape, and efficiently filters out higher undulator harmonics.

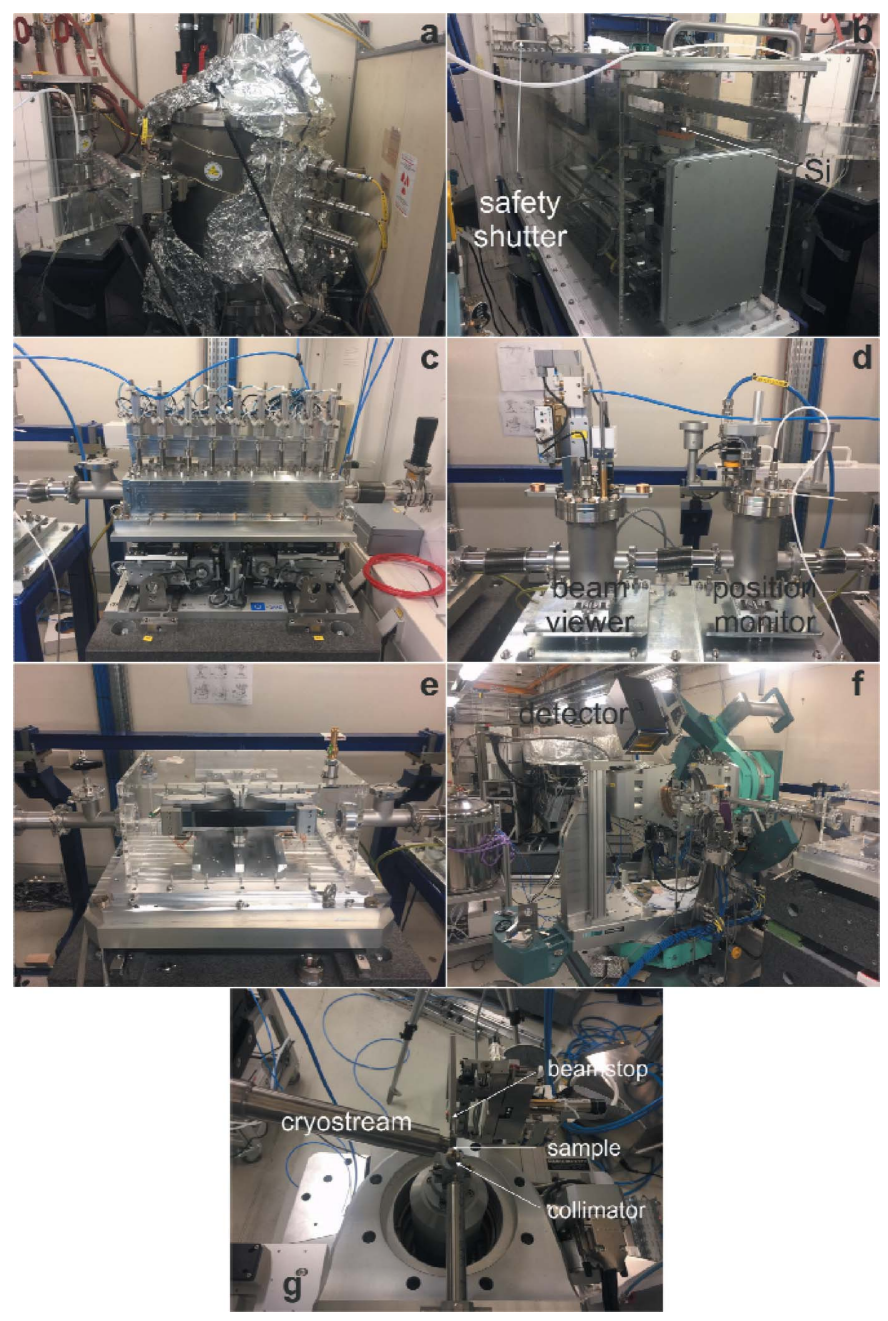

Figure 2

Photographs of the main components of side station. (a) The first monochromator chamber, $(b)$ the second monochromator chamber, (c) the transfocator, $(d)$ the beam-position monitor and beam viewer, (e) the multilayer mirror focusing horizontally, $(f)$ the five-axis diffractometer with detector, and $(g)$ the collimator, beamstop, sample and Cryostream.

The use of a transfocator with 1D lenses allows us to adjust the focal spot in the vertical direction in a range from 30 to $100 \mu \mathrm{m}$ FWHM. In combination with a fixed-geometry multilayer mirror for horizontal focusing, astigmatic imaging of the source to a circular spot becomes available. The estimated heat load is never higher than $10 \mathrm{~mW}$, which allows us to use the generic version of the monochromatic beam transfocator developed at the ESRF. The transfocator slots can be filled in a $1+2+4+8+16+32+64$ pattern with the Be lenses, ensuring the necessary flexibility. The horizontal opening of the lens is $2.8 \mathrm{~mm}$, thus intercepting essentially the full horizontal beam. The use of a multilayer (ML) mirror for the horizontal focusing assures full interception of the X-ray beam. Positioning at a distance of $1500 \mathrm{~mm}$ from the sample leaves enough space for complex sample environments when required, and for the beam-conditioning unit. The beam position and intensity are monitored with a diamond beamposition monitor and a beam viewer inserted between the ML 
mirror and transfocator. The performance of the station is summarized in Table 1.

Diffuse scattering signal collection requires the minimization of parasitic scattering from beamline elements and air scattering. The beam-conditioning unit comprises the following elements: beam-shaping slits (JJ X-ray Slit Systems), monochromatic beam absorber, collimator and beamstop. A removable collimator and beamstop with magnetic mounts were preferred. The goniostat, of Eulerian type, ensures freedom of sample rotations and holds the detector on the movable arm (gamma), with $2 \theta$ angles accessible up to $\sim 170^{\circ}$. A heavy-load translation stage for the pixel detector has been designed to provide a variable detector-to-sample distance from 150 to $400 \mathrm{~mm}$. The goniometer is equipped with a PILATUS3 $1 \mathrm{M}$ detector, which meets the stringent requirements for the quantitative measurement of diffuse scattering. The detector offers the following advantages: high stability, a large dynamic range, a narrow point spread function (1 pixel), a low spatial distortion and high sensitivity (quantum efficiency $50 \%$ at $17.8 \mathrm{keV}$ ) (Donath et al., 2013).

A growing set of auxiliary equipment is available to users. Currently, it contains a diamond-anvil-cell adapter for highpressure experiments, an Oxford Cryostream 700 nitrogen blower ( $80-500 \mathrm{~K})$, a helium closed-cycle cryostat (10-300 K) and a heat blower (300-1000 K) that have been employed already. Compact cells for the application of electric fields for temperatures up to $523 \mathrm{~K}$ can be used in conjunction with the standard $49 \mathrm{~mm}$ Huber gonio head.

\subsection{Control software}

The optics and motors are controlled by the standard ESRF IcePAPs and SPEC environment (Janvier et al., 2014). Recorded frames are saved in the CBF file format (Dectris, 2013). The diffractometer is controlled via the user-friendly program Pylatus previously developed on the SwissNorwegian Beamline of the ESRF (SNBL) (Dyadkin et al., 2016). The program allows synchronization of the motors and the detector and control of all the auxiliary equipment such as the heat blower, Cryostream and electric field cell. Different experimental configurations can be easily configured to set up a single-crystal or powder diffraction experiment. It is also possible to program long sequences with different temperatures and detector positions.

\subsection{Data analysis}

The amount of data collected in a diffraction or diffuse scattering experiment can be substantial. Data processing almost in real time is accessible on site, allowing on-the-fly optimization of the experiment. Typical collection rates amount to $\sim 0.1-1 \mathrm{~GB} \mathrm{~min}^{-1}$. This allows fast analysis of the diffraction results and optimization of the experiment strategy.

Several tools are available for users to process the collected diffraction data. One option is the use of the Rigaku/Oxford Diffraction CrysAlis PRO software (Agilent Technologies Ltd, Yarnton, Oxfordshire, UK) for peak harvesting and intensity integration. The $\mathrm{CBF}$ data have to be converted into
Table 1

Characteristics of the side station for the (311)-(422) double-crystal monochromator.
Source characteristics

Source size

Source divergence (electron)

Source divergence (photon)

ptics

Monochromator

Transfocator (Be-CRLs)

Multilayer mirror

Wavelengths available

Focused beam size

Detector system

Hybrid pixel detector

(PILATUS3 1M)
$402 \mu \mathrm{m}(\mathrm{H}) \times 7.9 \mu \mathrm{m}(\mathrm{V})$

$10.7 \mu \mathrm{rad}(\mathrm{H}) \times 3.2 \mu \mathrm{rad}(\mathrm{V})$

$12.4 \mathrm{keV}: 13.2 \mu \mathrm{rad}(\mathrm{H}) \times 8.4 \mu \mathrm{rad}(\mathrm{V})$

$17.8 \mathrm{keV}: 12.5 \mu \mathrm{rad}(\mathrm{H}) \times 7.2 \mu \mathrm{rad}(\mathrm{V})$

$24.8 \mathrm{keV}: 12.0 \mu \mathrm{rad}(\mathrm{H}) \times 6.36 \mu \mathrm{rad}(\mathrm{V})$

Double-crystal diamond(311)/silicon(422)

First crystal in vacuum, water cooled, Laue

Second crystal in helium atmosphere, no cooling, Bragg diffraction plane close to the horizontal, wavelengths $0.5-1.0 \AA$ Generic model, cylindrical lenses

Lens curvature radius $\infty(\mathrm{H}) \times 400 \mu \mathrm{m}(\mathrm{V})$

Geometry: $p=33.5 \mathrm{~m}, q=1.5 \mathrm{~m}$

Substrate length: $L=300 \mathrm{~mm}$

System: $\left[\mathrm{Pd} / \mathrm{B}_{4} \mathrm{C}\right]_{60} / \mathrm{Cr}$

$d$ spacing at centre: $4.0 \mathrm{~nm}$

Incidence angle: $0.373^{\circ}$ to $0.750^{\circ}$

$0.5226 \AA$ (equivalent of $121212 \mathrm{Si}$ )

$0.5701 \AA$ (equivalent of $111111 \mathrm{Si}$ )

$0.6968 \AA$ (equivalent of $999 \mathrm{Si}$ )

$0.7839 \AA$ (equivalent of $888 \mathrm{Si}$ )

$50 \mu \mathrm{m}(\mathrm{H}) \times 50 \mu \mathrm{m}(\mathrm{V})$

$450 \mu \mathrm{m}$ Si sensor thickness

Pixel size: $172 \times 172 \mu \mathrm{m}$

Point spread function: 1 pixel (FWHM)

Quantum efficiency: $50 \%$ at $17.8 \mathrm{keV}$ the ESPERANTO format first. CrysAlis PRO also offers the possiblity of reconstructing reciprocal-space maps from the collected data. Alternatively, reciprocal-space maps can be reconstructed with locally developed software directly from the CBF data, using the crystal orientation and geometry description provided by CrysAlis PRO. Fast inspection of the resulting maps is performed with the Albula software. Most of the functions of the SNBL Toolbox are also accessible (Dyadkin et al., 2016).

Although the diffractometer was primarily developed for single-crystal diffraction and diffuse scattering experiments, it is also used for powder diffraction. Data analysis typically commences with the use of DIOPTAS software (Prescher \& Prakapenka, 2015), which allows on-the-fly data processing and rapid exploration of two-dimensional X-ray diffraction area-detector data. It is also possible for users to use another processing tool for powder data, bubble (Dyadkin et al., 2016), developed on the SNBL beamline, based on the use of the pyFAI library for azimuthal integration of the raw images (Kieffer \& Karkoulis, 2013).

The side station is mainly used for diffuse scattering experiments. In order to interpret the results of such experiments correctly, it is often necessary to compare the experimental results with those of simulations. For this purpose, various locally developed open-source software packages are available. $a b 2 t d s$ is an open-source package that allows, among 
other things, modelling of the phonon contribution to the diffuse scattering (Wehinger \& Mirone, 2013). It is a postprocessing tool which takes dynamic matrices computed by an external program [e.g. CASTEP (Clark et al., 2005) or phonopy (Togo \& Tanaka, 2015) which can be used in conjunction with VASP (Kresse \& Furthmüller, 1996), Wien2K (Blaha et al., 2001), abinit (Gonze et al., 2009) and SIESTA (Soler et al., 2002)] as input, and produces TDS maps that can be compared directly with the experimental results.

\subsection{Performance}

2.4.1. Resolution function. The resolution of the instrument depends mainly on the sample-to-detector distance, beam size, detector pixel size and sample properties (crystallinity). We have characterized the resolution of the side station using high-quality standard $\mathrm{LaB}_{6}$ powder. We present here two measurements carried out with two different sample-todetector distances that are commonly used: $d=414 \mathrm{~mm}$ and $d=244 \mathrm{~mm}$, with a beam size of $40 \mu \mathrm{m} \times 20 \mu \mathrm{m}$ FWHM and

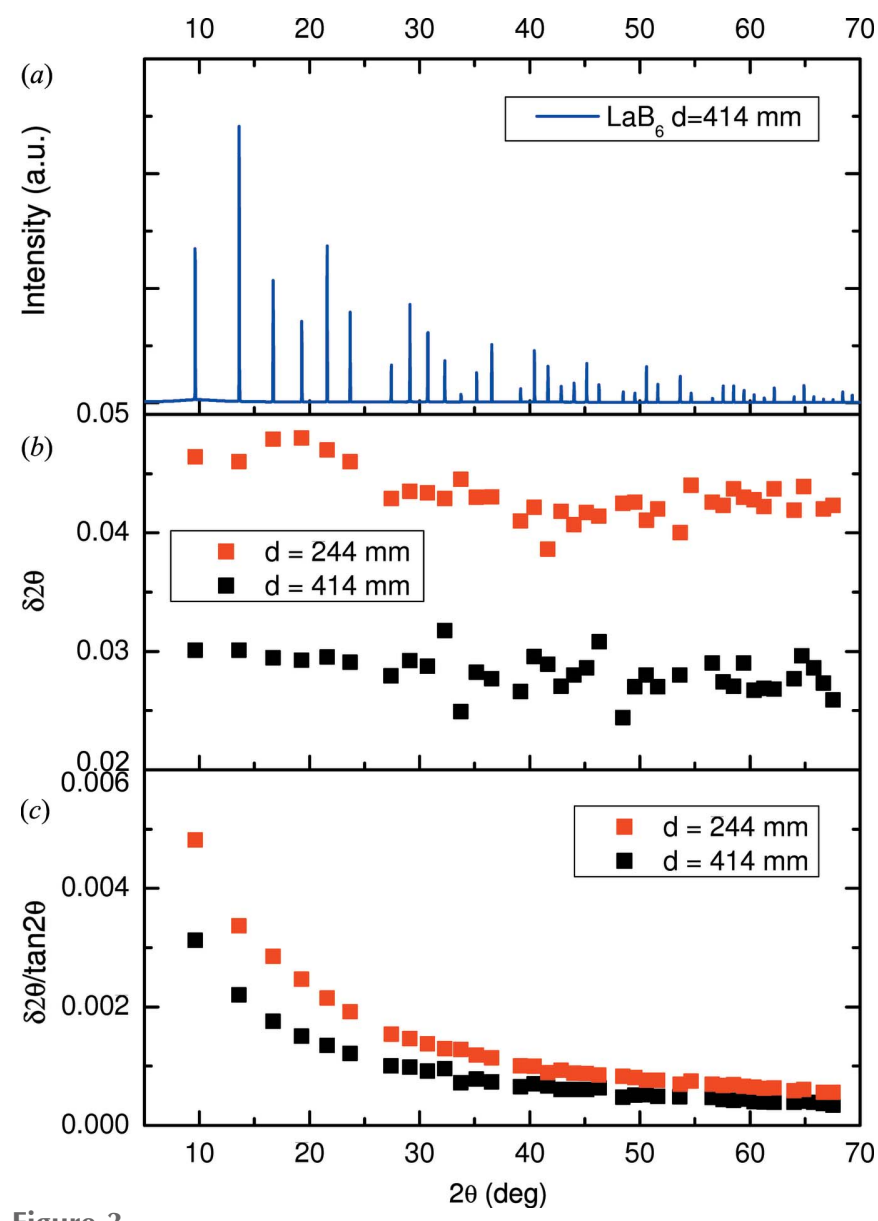

Figure 3

(a) Powder diffraction pattern from $\mathrm{LaB}_{6}$ powder measured with a large sample-to-detector distance $d=414 \mathrm{~mm}$. (b) A plot of $\delta 2 \theta$ versus $2 \theta$, representing the experimental resolution function. (c) A plot of $\delta 2 \theta / \tan 2 \theta$ versus $2 \theta$. The data were measured from $\mathrm{LaB}_{6}$ powder in a $100 \mu \mathrm{m}$ capillary rotated over $60^{\circ}$ with two sample-to-detector distances of $d=414 \mathrm{~mm}$ (black) and $244 \mathrm{~mm}$ (red), a beam size of approximately $50 \mu \mathrm{m} \times 50 \mu \mathrm{m}$ and $17.794 \mathrm{keV}$ energy.
Table 2

Experimental details for the alum data set.

\begin{tabular}{ll}
\hline Crystal data & \\
Chemical formula & $\mathrm{AlH}_{24} \mathrm{KO}_{20} \mathrm{~S}_{2}$ \\
Space group & $P a \overline{3}$ \\
$a(\AA)$ & 12.1892 \\
$b(\AA)$ & 12.1892 \\
$c(\AA)$ & 12.1892 \\
Wavelength $(\AA)$ & 0.5226 \\
Temperature (K) & 293 \\
$Z$ & 4 \\
& \\
Data collection & 5278 \\
No. of measured reflections & 716 \\
No. of independent reflections & 687 \\
No. of reflections with $I>2 \sigma(I)$ & 0.059 \\
$R_{\text {int }}$ & $\theta_{\min }=2.5^{\circ}, \theta_{\max }=20.5^{\circ}$ \\
Angular range & $-15 \rightarrow 8$ \\
$h$ & $-13 \rightarrow 16$ \\
$k$ & $-9 \rightarrow 14$ \\
$l$ & \\
Refinement on $F^{2}$ & \\
$R\left[F^{2}>2 \sigma\left(F^{2}\right)\right]$ & 0.0395 \\
No. of reflections used in refinement & 716 \\
No. of parameters used & 68 \\
Extinction coefficient & 0.171 \\
\hline
\end{tabular}

17.794 keV energy. Fig. 3(a) shows the powder diffraction pattern from $\mathrm{LaB}_{6}$ powder measured with a large sample-todetector distance of $d=414 \mathrm{~mm}$, obtained by assembling three data sets measured with three detector-angle setups $\left(\Gamma=12^{\circ}\right.$, $32^{\circ}$ and $48^{\circ}$, where $\Gamma$ is the vertical angle of the detector with respect to the incoming beam). In Figs. $3(b)$ and $3(c)$ we also plot $\delta 2 \theta$ versus $2 \theta$ and $\delta 2 \theta / \tan 2 \theta$ versus $2 \theta$, respectively, derived from the powder patterns. Here, two data sets $\left(\Gamma=19^{\circ}\right.$ and $\left.48^{\circ}\right)$ and three data sets $\left(\Gamma=12^{\circ}, 32^{\circ}\right.$ and $\left.54^{\circ}\right)$ were measured for $d=244 \mathrm{~mm}$ and $d=414 \mathrm{~mm}$, respectively. These results demonstrate that the achieved resolution of the side station $(\delta 2 \theta \leq 0.05)$ is as good as the values obtained in other modern dedicated powder diffraction synchrotron beamlines (Murray et al., 2017).

2.4.2. Structure solution from single-crystal diffraction. The capabilities of the side station with respect to data collection for structure refinements from single-crystal diffraction data have been evaluated by solving the crystal structure of an alum crystal at ambient temperature with a wavelength of $0.5226 \AA$ and a detector distance of $d=244 \mathrm{~mm}$. A total of 720 frames were recorded $\left(0.5^{\circ}\right.$ step with $0.5 \mathrm{~s}$ counting time) with a single detector-angle setup $\left(\Gamma=19^{\circ}\right)$. A total of 5278 reflections were integrated in the angular range 2.5-20.5 using the Crys Alis PRO software, yielding intensities for 716 independent reflections. The structure determination was carried out with the SHELX package (Sheldrick, 2015) and was refined with $R=0.0395$ (see Table 2 for details). Fig. 4 shows a graphical rendering of the resulting structure produced with VESTA (Momma \& Izumi, 2011).

When measuring a diffraction data set with the view of solving the crystal structure using a single-photon-counting detector, special care should be paid to the dynamic range of the detector to avoid overexposure of the pixels. Such a situation can be avoided by choosing an appropriate 
(a)
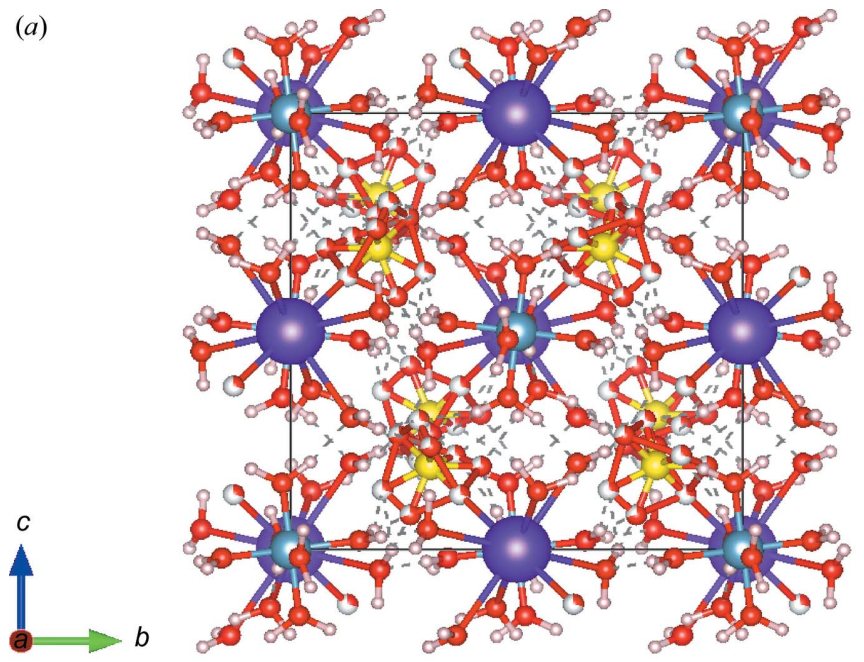

(b)

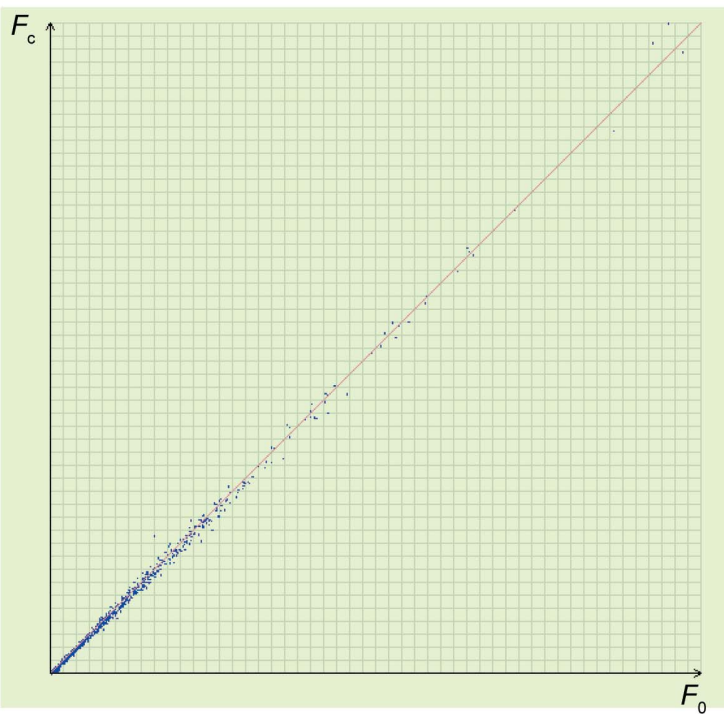

Figure 4

(a) The alum structure as refined from SHELX sofware. (b) $F_{\mathrm{c}}$ versus $F_{\mathrm{o}}$ plot, where $F_{\mathrm{c}}$ and $F_{\mathrm{o}}$ are the calculated and experimental intensities, respectively. Each dot represents one measured reflection.

attenuator to avoid overexposure of strong reflections and overattenuation of weak ones. The $F_{\mathrm{c}}-F_{\mathrm{o}}$ plot is linear, as illustrated in Fig. 4(b) for the case of alum.

2.4.3. Diffuse scattering example: lattice dynamics survey in reciprocal space. One of the main purposes of the side station is to provide an overview in reciprocal space of the lattice dynamics of a single crystal, in order to guide subsequent energy- and momentum-resolved phonon experiments with the high-resolution IXS spectrometer. Here we present an example of TDS mapping obtained at room temperature with a $\mathrm{Bi}_{2} \mathrm{SiO}_{5}$ (BSO) single crystal (Fig. 5). BSO is ferroelectric at room temperature, and the paraelectric-ferroelectric transition is driven by the freezing of the ferroelectric soft mode at the $\Gamma$ point. In addition to the ferroelectric instability, an antiferroelectric instability also occurs due to the softening of a transverse optic (TO) phonon at the Y point of the Brillouin zone edge. This additional softening induces a (a) $H 1 L$ plane - experiment

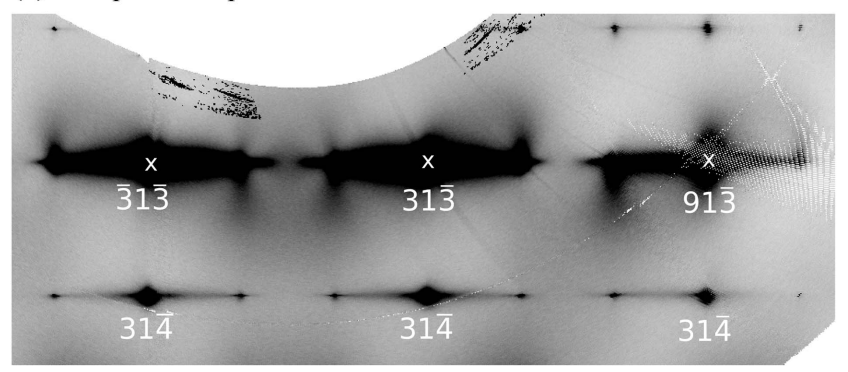

(b) DFT

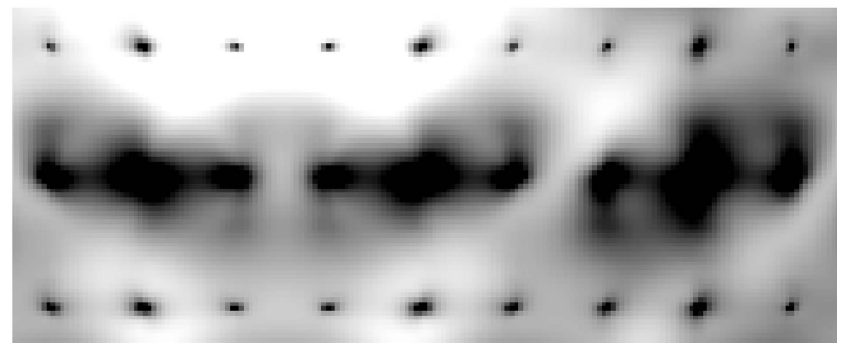

Figure 5

(a) An example of a reciprocal-space map reconstruction obtained with

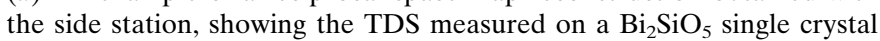
in the ferroelectric phase. The intensity distribution in the $H 1 L$ plane measured under room-temperature conditions shows shuttle-like TDS features along the [100] direction produced by a low-lying transverse acoustic branch due to TO-TA branch repulsion (see main text). (b) The corresponding map as calculated from DFT.

suppression of the transverse acoustic (TA) frequency, likely driven by TO-TA mode repulsion. The supression of the TA frequency produces characteristic shuttle-like TDS patterns oriented along the [100] direction [see the $H 1 L$ plane reconstruction in Fig. 5(a)] that can be used to determine the Brillouin zone relevant for the IXS measurement. The experimental data can then be compared with theoretical TDS maps calculated using ab2tds (Wehinger \& Mirone, 2013), where the phonons were previously calculated using density functional theory (DFT) [Fig. 5(b)]. Here, good agreement is obtained with the theoretical TDS map and the anisotropic TDS features are well reproduced, confirming the inelastic nature of the diffuse signal.

A second example is presented in Fig. 6, which shows the reconstructed reciprocal-space maps of lead stannate, $\mathrm{Pb}_{2} \mathrm{SnO}_{4}$, in the $0 K L$ and $H K 0$ planes. In this case, a lowenergy TA branch produces characteristic elongated TDS features in the $H K 0$ plane, most pronounced close to the strongest Bragg peaks where acoustic phonons have a stronger intensity. The characteristic diffuse features belong to the $H K 0$ plane perpendicular to the chains of edge-sharing $\mathrm{SnO}_{6}$ octahedral units along the $c$ axis, and are not affected by the orthorhombic-tetragonal phase transition occurring at $T \simeq$ $350 \mathrm{~K}$. They are well reproduced by the DFT calculations and absent in the $0 K L$ plane, in the direction parallel to the chains. This example shows that TDS is well suited, through the investigation of lattice dynamics in reciprocal space, to studying anisotropic effects that can be carried out together with structural investigations. 
(a)
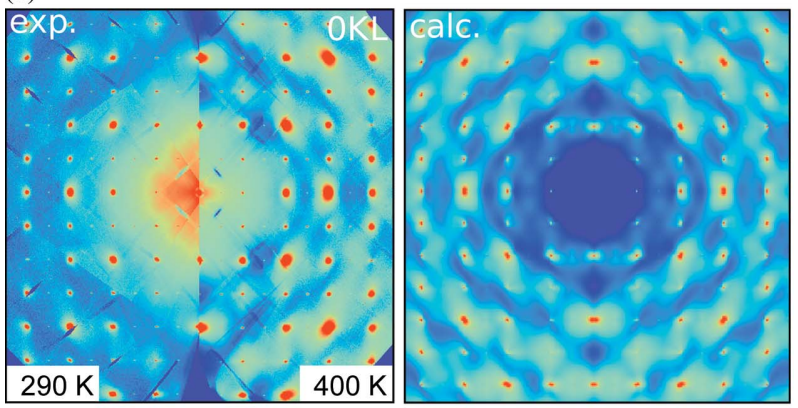

(b)
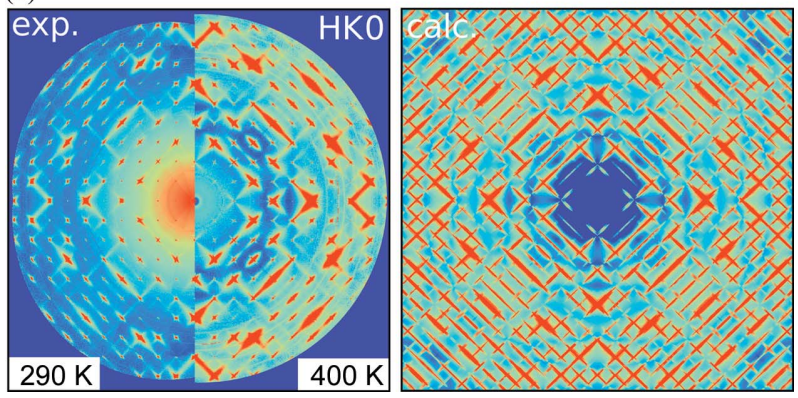

Figure 6

Reciprocal-space maps of $\mathrm{Pb}_{2} \mathrm{SnO}_{4}$ in $(a)$ the $0 K L$ and $(b)$ the $H K 0$ planes. The left-hand panels correspond to the measured TDS in the orthorhombic $(T=290 \mathrm{~K})$ and tetragonal $(T=400 \mathrm{~K})$ phases, and the right-hand panels to the theoretical maps calculated using DFT.

\section{Conclusions}

A new diffractometer has been built as a side station on the ID28 beamline at the ESRF. The instrument is primarily dedicated to the study of diffuse scattering in a large class of materials, ranging from strongly correlated electron systems to nanoscale-modulated and low-dimensional systems, and may constitute a powerful tool in the study of lattice dynamics complementing inelastic X-ray scattering studies. Investigations of a large class of crystalline systems with correlated disorder will greatly benefit from the dedicated station, thanks to a flexible sample environment. The high brilliance of the $\mathrm{X}$-ray source, coupled with state-of-the-art detection schemes using a hybrid pixel detector, will open the window to timeresolved studies and studies under extreme conditions of pressure and temperature. The diffuse scattering station, in combination with the existing IXS instrument on ID28, constitutes a globally unique experimental station, offering unprecedented capabilities in the study of lattice dynamics in condensed matter and of the real structure of functional materials to the large ESRF user community.

\section{Acknowledgements}

Fruitfull discussion with Dmitry Chernyshov are gratefully acknowledged.

\section{Funding information}

This study was supported by the BMBF project 05K13RF1 and 05K13RF2 and a joint DFG-ANR project WI1232/41-1.

\section{References}

Antonangeli, D., Krisch, M., Farber, D. L., Ruddle, D. G. \& Fiquet, G. (2008). Phys. Rev. Lett. 100, 085501.

Antonangeli, D., Siebert, J., Aracne, C. M., Farber, D. L., Bosak, A., Hoesch, M., Krisch, M., Ryerson, F. J., Fiquet, G. \& Badro, J. (2011). Science, 331, 64-67.

Antonangeli, D., Siebert, J., Badro, J., Farber, D. L., Fiquet, G., Morard, G. \& Ryerson, F. J. (2010). Earth Planet. Sci. Lett. 295, 292296.

Astuto, M. d', Yamada, I., Giura, P., Paulatto, L., Gauzzi, A., Hoesch, M., Krisch, M., Azuma, M. \& Takano, M. (2013). Phys. Rev. B, 88, 014522.

Bencivenga, F., Cunsolo, A., Krisch, M., Monaco, G., Orsingher, L., Ruocco, G., Sette, F. \& Vispa, A. (2007). Phys. Rev. Lett. 98, 085501.

Blaha, K. S. P., Schwarz, K., Madsen, G., Kvasnicka, D. \& Luitz, J. (2001). WIEN2k: An Augmented Plane Wave plus Local Orbitals Program for Calculating Crystal Properties. Technische Universität Wien, Wien, Austria.

Borissenko, E., Goffinet, M., Bosak, A., Rovillain, P., Cazayous, M., Colson, D., Ghosez, P. \& Krisch, M. (2013). J. Phys. Condens. Matter, 25, 102201.

Bosak, A., Chernyshov, D., Wehinger, B., Winkler, B., Le Tacon, M. \& Krisch, M. (2015). J. Phys. D Appl. Phys. 48, 504003.

Clark, S. J., Segall, M. D., Pickard, C. J., Hasnip, P. J., Probert, M. I. J., Refson, K. \& Payne, M. C. (2005). Z. Kristallogr. 220, 567-570.

Damart, T., Giordano, V. M. \& Tanguy, A. (2015). Phys. Rev. B, 92 , 094201.

Dectris (2013). Pilatus User Manual. Dectris Ltd, Switzerland. https:// www.dectris.com.

Donath, T., Brandstetter, S., Cibik, L., Commichau, S., Hofer, P., Krumrey, M., Lüthi, B., Marggraf, S., Müller, P., Schneebeli, M., Schulze-Briese, C. \& Wernecke, J. (2013). J. Phys. Conf. Ser. 425, 062001.

Dyadkin, V., Pattison, P., Dmitriev, V. \& Chernyshov, D. (2016). J. Synchrotron Rad. 23, 825-829.

Fiquet, G., Badro, J., Gregoryanz, E., Fei, Y. \& Occelli, F. (2009). Phys. Earth Planet. Inter. 172, 125-129.

Gonze, X., Amadon, B., Anglade, P.-M., Beuken, J.-M., Bottin, F., Boulanger, P., Bruneval, F., Caliste, D., Caracas, R., Côté, M., Deutsch, T., Genovese, L., Ghosez, P., Giantomassi, M., Goedecker, S., Hamann, D. R., Hermet, P., Jollet, F., Jomard, G., Leroux, S., Mancini, M., Mazevet, S., Oliveira, M. J. T., Onida, G., Pouillon, Y., Rangel, T., Rignanese, G.-M., Sangalli, D., Shaltaf, R., Torrent, M., Verstraete, M. J., Zerah, G. \& Zwanziger, J. W. (2009). Comput. Phys. Commun. 180, 2582-2615.

Gvasaliya, S. N., Cervellino, A., Roessli, B., Rotaru, G. M., Cowley, R. A., Lushnikov, S. G., Shaplygina, T. A. \& Bouchenoire, L. (2012). J. Phys. Condens. Matter, 24, 455401.

Hoesch, M., Bosak, A., Chernyshov, D., Berger, H. \& Krisch, M. (2009). Phys. Rev. Lett. 102, 086402.

Izzo, M. G., Bencivenga, F., Gessini, A., Cunsolo, A. \& Masciovecchio, C. (2011). Philos. Mag. 91, 1767-1775.

Janvier, N., Clement, J. M., Fajardo, P. \& Cuní, G. (2014). Proceedings of the 14th International Conference on Accelerator and Large Experimental Physics Control Systems (ICALEPCS 2013), 6-11 October 2013, San Francisco, California, USA, pp. 766-769, TUPPC081. Geneva: JACoW.

Kieffer, J. \& Karkoulis, D. (2013). J. Phys. Conf. Ser. 425, 202012.

Kresse, G. \& Furthmüller, J. (1996). Phys. Rev. B, 54, 11169-11186.

Leroux, M., Errea, I., Le Tacon, M., Souliou, S. M., Garbarino, G., Cario, L., Bosak, A., Mauri, F., Calandra, M. \& Rodière, P. (2015). Phys. Rev. B, 92, 140303.

Le Tacon, M., Bosak, A., Souliou, S. M., Dellea, G., Loew, T., Heid, R., Bohnen, K.-P., Ghiringhelli, G., Krisch, M. \& Keimer, B. (2013). Nat. Phys. 10, 52-58.

Lethbridge, Z. A. D., Walton, R. I., Bosak, A. \& Krisch, M. (2009). Chem. Phys. Lett. 471, 286-289. 
Lortz, R., Viennois, R., Petrovic, A., Wang, Y., Toulemonde, P., Meingast, C., Koza, M. M., Mutka, H., Bossak, A. \& Miguel, A. S. (2008). Phys. Rev. B, 77, 224507.

Momma, K. \& Izumi, F. (2011). J. Appl. Cryst. 44, 1272-1276.

Murray, C. A., Potter, J., Day, S. J., Baker, A. R., Thompson, S. P., Kelly, J., Morris, C. G., Yang, S. \& Tang, C. C. (2017). J. Appl. Cryst. 50, 172-183.

Niss, K., Dalle-Ferrier, C., Giordano, V. M., Monaco, G., Frick, B. \& Alba-Simionesco, C. (2008). J. Chem. Phys. 129, 194513.

Pogna, E. A. A., Rodríguez-Tinoco, C., Cerullo, G., Ferrante, C., Rodríguez-Viejo, J. \& Scopigno, T. (2015). Proc. Natl Acad. Sci. USA, 112, 2331-2336.

Prescher, C. \& Prakapenka, V. B. (2015). High. Press. Res. 35, $223-$ 230.

Rueff, J. P., Krisch, M. \& Lorenzen, M. (2002). High. Press. Res. 22, 53-56.

Sheldrick, G. M. (2015). Acta Cryst. A71, 3-8.

Soler, J. M., Artacho, E., Gale, J., García, A., Junquera, J., Ordejón, P. \& Sánchez-Portal, D. (2002). J. Phys. Condens. Matter, 14, $2745-$ 2779.

Souliou, S. M., Li, Y., Du, X., Le Tacon, M. \& Bosak, A. (2016). Phys. Rev. B, 94, 184309.
Togo, A. \& Tanaka, I. (2015). Scr. Mater. 108(Suppl.C), 1-5.

Walters, A. C., Walker, H. C., Springell, R., Krisch, M., Bosak, A., Hill, A. H., Zvorişte-Walters, C. E., Colineau, E., Griveau, J. C., Bouëxière, D., Eloirdi, R., Caciuffo, R. \& Klimczuk, T. (2015). J. Phys. Condens. Matter, 27, 325702.

Wehinger, B., Bosak, A., Piccolboni, G., Refson, K., Chernyshov, D. I., Ivanov, A., Rumiantsev, A. \& Krisch, M. (2014). J. Phys. Condens. Matter, 26, 115401.

Wehinger, B., Bosak, A., Refson, K., Mirone, A., Chumakov, A. \& Krisch, M. (2015). J. Phys. Condens. Matter, 27, 305401.

Wehinger, B., Chernyshov, D., Krisch, M., Bulat, S., Ezhov, V. \& Bosak, A. (2014). J. Phys. Condens. Matter, 26, 265401.

Wehinger, B. \& Mirone, A. (2013). ab2tds's Documentation. http:// ftp.esrf.fr/scisoft/AB2TDS/index.html.

Welberry, T. R. (1985). Rep. Progr. Phys. 48, 1543.

Weng, S.-C., Xu, R., Said, A. H., Leu, B. M., Ding, Y., Hong, H., Fang, X., Chou, M. Y., Bosak, A., Abbamonte, P., Cooper, S. L., Fradkin, E., Chang, S. \& Chiang, T. (2014). Europhys. Lett. 107, 36006.

Zocco, D. A., Krannich, S., Heid, R., Bohnen, K.-P., Wolf, T., Forrest, T., Bosak, A. \& Weber, F. (2015). Phys. Rev. B, 92, 220504. 